\title{
超声促进两组分多彩发光体系分子聚集体的共组装 及其对酸的响应行为
}

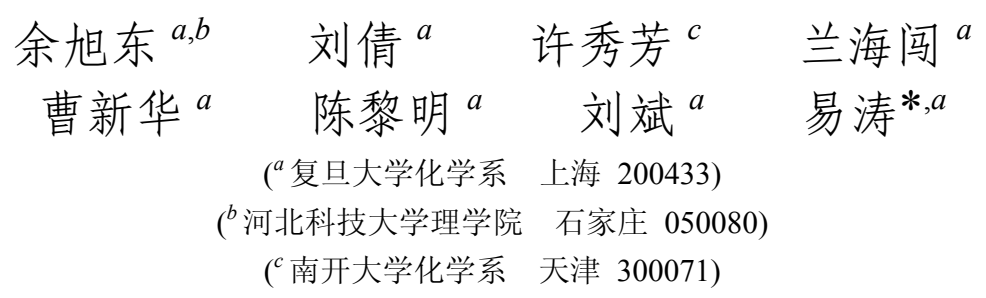

\begin{abstract}
摘要 设计合成了一种含凝胶因子和微晶的两组分凝胶体系, 由发蓝光的氨基酸衍生物和发红光的范唑衍生物构成. 在超声和热的协同作用下，可促进凝胶因子的羧基和微晶分子中的苊唑氮进行酸碱配位，从而实现该两组分在分子聚 集体层次的共组装. 两种化合物分别能发出蓝色和红色荧光, 便于我们通过共聚焦显微镜(CLSM)来直接观测其微观聚 集行为. CLSM 图片表明通过加热冷却的方法所获得的干凝胶其微观结构是由发红光的棒状微晶和发蓝光的纤维组成 的混合体; 当通过一个预超声再加热-冷却的过程, 可以得到一种均匀的同时发射蓝光和红光的凝胶纤维聚集体. 结果 表明，超声和热的协同作用可以有效地促进分子间的聚集，从而形成均相体系. 进一步，我们通过超声诱导的分子间 共聚集构建了微纳米层次上的多彩发光体系. 该体系发射光谱可以通过两种成分的混合比例来精确调控，同时可以通 过加入质子酸进一步调控. 与此同时, 酸的加入也诱导了两组分组装体发生由纤维向囊泡的形貌变化, 并伴随着凝胶 向溶胶的宏观相态转变. 当加入碱后, 该溶胶可恢复为凝胶. 这种具有多重功能并易于调控的两组分凝胶在可视化分 子识别、控制缓释、刺激响应和记忆材料等领域具有潜在应用价值.
\end{abstract}

关键词 两组分凝胶; 超声; 共组装; 苂光; 酸响应

\section{Ultrasound Assisted Co-aggregation of a Two-component System with Multicolor Emission and Its Response to Acid}

\author{
Yu, Xudong ${ }^{a, b} \quad{\text { Liu, } \text { Qian }^{a} \quad \mathrm{Xu}, \text { Xiufang }^{c} \quad \text { Lan, Haichuang }}^{a}$ \\ Cao, Xinhua ${ }^{a} \quad$ Chen, Liming $^{a} \quad$ Liu, Bin $^{a} \quad$ Yi, Tao*,a \\ ( ${ }^{a}$ Department of Chemistry, Fudan University, Shanghai 200433) \\ $\left({ }^{b}\right.$ College of Science, Hebei University of Science and Technology, Shijiazhuang 050080) \\ ( ${ }^{c}$ Department of Chemistry, Nankai University, Tianjin 300071)
}

\begin{abstract}
In this paper, a two-component gel/micro-crystal system including amino acid and imidazole-based derivatives with blue and red emission, respectively, is designed and obtained. The two emission colors are convenient for the study of the co-aggregation of the two molecules via double channel confocal laser scanning microscopy. The two-component system could response to ultrasound with the morphology change from the mixture of club-shaped microcrystals and helical fibers by a thermal process to homogenous fibers exposing to sonication. Further evidence for the co-aggregation of the two components was obtained from circular dichromism (CD), IR spectra and X-ray powder diffraction. The self-assembly process between the two components was also studied by optimized molecular geometry calculation. Moreover, the co-aggregation obtained by the sonication then heating-cooling process, could not be destroyed by repeated heating-cooling. The gel-sol process could be further repeated for many times, suggesting that the co-aggregated gel was thermal-stable. It can be deduced that sonication weakened the intermolecular interaction between congeneric molecules and promoted the intermolecular hydrogen bonding between the two components. The results indicate that cooperation of the sonication and thermal processes is an effective way to prohibit phase separation and to promote gelation in the gel/micro-crystal system. Thus, a light harvest system is achieved by co-aggregation of the two components at nanoscale by means of sonication. The emission color of these two-component gels can be tuned by changing the ratio of the two compounds. These gels are sensitive to acid, giving
\end{abstract}

* E-mail: yitao@fudan.edu.cn; Tel.: 021-55664330; Fax: 021-55664621

Received June 1, 2012; published July 13, 2012.

Supporting information for this article is available free of charge via the Internet at http://sioc-journal.cn.

Project supported by the National Natural Science Foundation of China (Nos. 91022021 and 30890141), the National Basic Research Program of China (No. 2009CB930400), the Program for Innovative Research Team in University (No. IRT1117) and the Shanghai Leading Academic Discipline Project (No. B108). 项目受国家自然科学基金(Nos. 91022021 and 30890141)、973 项目(No. 2009CB930400)、教育部创新团队(No. IRT1117)和上海市重点学科(No. B108) 资助. 
clear spectral variation, accompanied by a gel to sol transition. The morphology of the two-component system is changed from a fiber structure to vesicles without phase separation when responding to acid and metal ions. Moreover, both the gel state and the morphology can be reversed by further addition of alkali. This kind of multifunctional and tunable two-component gel should have potential applications on the fields of visible molecular recognition, controllable release, stimulus responsive and memory materials.

Keywords two-component gel; ultrasound; co-aggregation; fluorescence; acid response

\section{1 引言}

近年来，在超分子领域，基于主客体相互作用(例 如氢键或酸碱作用 ${ }^{[1]}$ 、静电作用 ${ }^{[2]} 、 \pi-\pi$ 相互作用 ${ }^{[3]}$ 、金 属配位 ${ }^{[4]}$ 及电荷转移作用 ${ }^{[5]}$ 等)的两组分体系倍受人们 关注. 这些两组分体系在形貌、手性、光学性质及刺激 响应等方面往往能展示出多重的功能化应用 ${ }^{[6]}$. 通过对 两组分比例的调控, 可以很容易地实现对这些功能的进 一步精确控制. 在凝胶等超分子聚集体中, 这种分子间 的主客体相互作用可以得到进一步放大. 但是在包含凝 胶-微晶的两组分体系中, 晶化和成胶过程的同时存在 经常会抑制两组分聚集体的共组装, 由于同种分子间极 强的自组装行为, 往往会导致相分离态的存在, 而得不 到均一的聚集体 ${ }^{[7]}$. 迄今为止, 这个问题并未得到广泛 的关注和良好的解决.

近几年来, 人们发现超声作为一种外界刺激因素可 以用来促进凝胶的形成 ${ }^{[8]}$. 这种声响应的小分子凝胶在 生物成像、药物控释和自适应系统等领域存在着广泛的 潜在应用. 超声的本质是通过高频率的压力波产生的空 化效应对材料产生物理或化学作用. 在卤代烃降解 ${ }^{[9]}$ 、 聚合反应 ${ }^{[10]}$ 、氧化还原反应 ${ }^{[1]}$ 、反应选择性 ${ }^{[12]}$ 等领域中, 这种空化作用已被人们广泛研究. 近年来, 人们发现超 声可以用来切割分子内氢键或 $\pi-\pi$ 相互作用, 使凝胶因 子由分子内聚集变为分子间聚集, 从而锁住溶剂, 限制 液体的流动, 使之凝胶化 ${ }^{[9,13,14]}$.

随着两组分体系及智能响应材料研究的深入, 我们 推断超声可以用来促进异种分子间的聚集组装, 从而在 更大范围内获得易调控的均相凝胶体系, 并同时具备两 种组分的各种功能 ${ }^{[15]}$. 因此, 在本工作中, 我们选用了 一种可发生氢键相互作用的凝胶-微晶体系作为研究对 象, 研究超声和热作为外界刺激因素在该体系中的功能 化表达. 胺基戊二酸 $(\mathbf{1})$ 在极性溶剂如醇类, DMSO, DMF 等溶剂中都能形成良好的凝胶. 分子 1 中裸露的羧 基可以用来和其他含 $\mathrm{N}$ 或 $\mathrm{O}$ 的官能团通过氢键进行配 位, 从而包结合适的有机分子. 在前面的报道中, 我们
发现分子 1 的水凝胶可以用来包结药物盐酸四环素, 并 进行缓释 ${ }^{[16]}$. 本工作中, 我们发现分子 1 在 DMSO 或 DMF 中形成的凝胶可发射蓝色荧光, 最大发射峰在 471 nm. 2,4,5-三取代的范坐衍生物可发出红色甚至近红外 的苂光, 范唑氮可被酸质子化, 从而进一步改变其发光 波长或发光强度. 在生物成像领域, 这种红光或近红外 发光具有较高的穿透度及较低的生物背景干扰 ${ }^{[17]}$. Weiss 课题组 ${ }^{[18]}$ 早在 1952 年就报道了该类分子的合成, 但是这类化合物的性质并未引起人们的注意. 在本文 中, 我们报道了分子 $\mathbf{1}$ 和 $\mathbf{2}$ 在超声和热协同作用下被诱 导产生的一种可逆的分子共组装体(Eq. 1). 该共组装体 还可以进一步对酸做出响应, 诱导其从纤维向囊泡的形 貌转变, 并伴随着宏观上凝胶-溶胶的相态转变(图 1); 该体系可以通过进一步引入三乙胺等碱性化合物，重新 恢复至凝胶态, 实现该过程的可逆性.

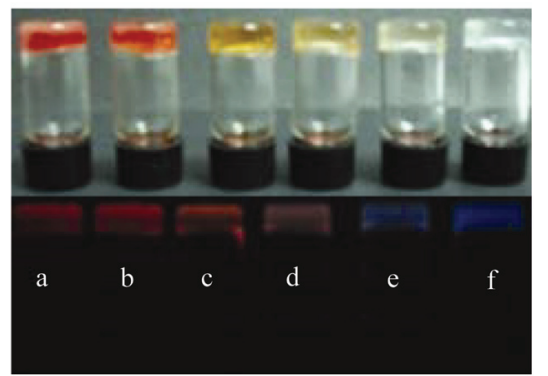

图 11 和不同比例 $1+2$ 的凝胶图片

Figure 1 Images of gel $\mathbf{1}$ and gels $\mathbf{1}+\mathbf{2}$ with different molar ratios of $\mathbf{1}$ and 2

up: bright field; below: dark; $\lambda_{\mathrm{ex}}=365 \mathrm{~nm}$. (a) $1: 1$, (b) $1: 0.66$, (c) $1: 0.18$, (d) $1: 0.09$, (e) $1: 0.02$, (f) only $1 ; c_{1}=12.5 \mathrm{mg} \cdot \mathrm{mL}^{-1}\left(1.29 \times 10^{-2} \mathrm{~mol} \cdot \mathrm{L}^{-1}\right)$

\section{2 结果与讨论}

\section{1 分子 1 和 2 化合物不同形态下的荧光发射}

在较低的浓度下, 分子 $\mathbf{1}$ 的 DMSO 溶液具有较弱的 蓝色荧光发射 $\left(5.0 \times 10^{-4} \mathrm{~mol} \cdot \mathrm{L}^{-1}, \lambda_{\mathrm{ex}}=377 \mathrm{~nm}\right.$, 图 2a), 在 $7.8 \times 10^{-6} \sim 1.0 \times 10^{-3} \mathrm{~mol} \cdot \mathrm{L}^{-1}$ 的浓度范围中, 该溶液 的荧光强度随着浓度的增加而增加(辅助材料图 S1). 在

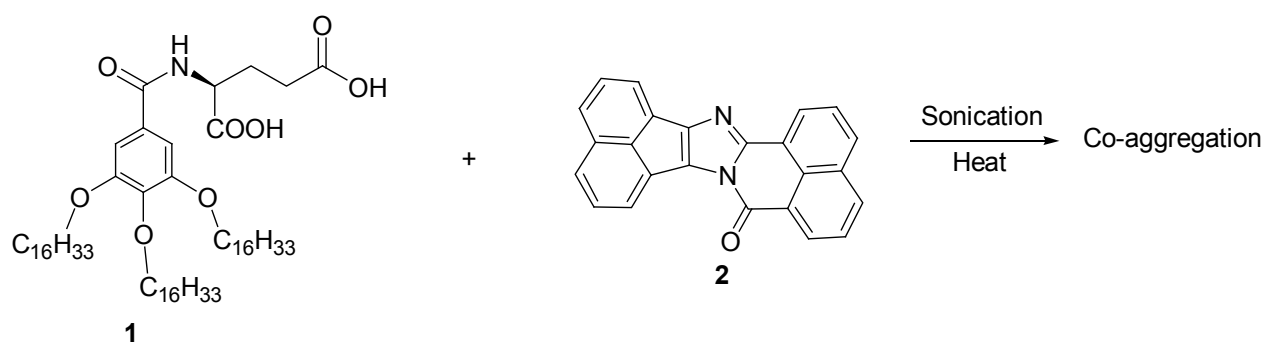


低浓度下 $\left(5.0 \times 10^{-4} \mathrm{~mol} \cdot \mathrm{L}^{-1}\right), 1$ 的 DMSO 溶液的苂光寿 命为 $1.3 \mathrm{~ns}(100 \%)$, 在饱和溶液下 $\left(1 \times 10^{-3} \mathrm{~mol} \cdot \mathrm{L}^{-1}\right), \mathbf{1}$ 分子间的聚集使其产生了一个较长的荧光寿命 $(8.9 \mathrm{~ns}$, $45.9 \%$, 辅助材料图 S2). 2 的 DMSO 溶液的最大发射峰 也随浓度增大发生了较大的红移(在 $1.1 \times 10^{-5} \mathrm{~mol} \cdot \mathrm{L}^{-1}$ 时发射为 $618 \mathrm{~nm}$; 在饱和溶液浓度下发射峰红移至 624 $\mathrm{nm}$, 图 $2 \mathrm{~b}$ 和辅助材料图 S3). 而 2 的固体粉末的最大发 射峰红移至 $639 \mathrm{~nm}$, 这些结果表明当浓度较高时, 2 分 子间具有较强的 $\pi \cdots \pi$ 堆积作用. 2 的饱和溶液的荧光寿 命为 $10.3 \mathrm{~ns}$ (辅助材料图 S4). 因此通过不同的激发与 发射波长的荧光信号收集, 可以很容易地在荧光共聚焦 显微镜(CLSM)下分别观测到 1 和 2 分子的聚集行为, 并 进一步研究它们的组装行为.
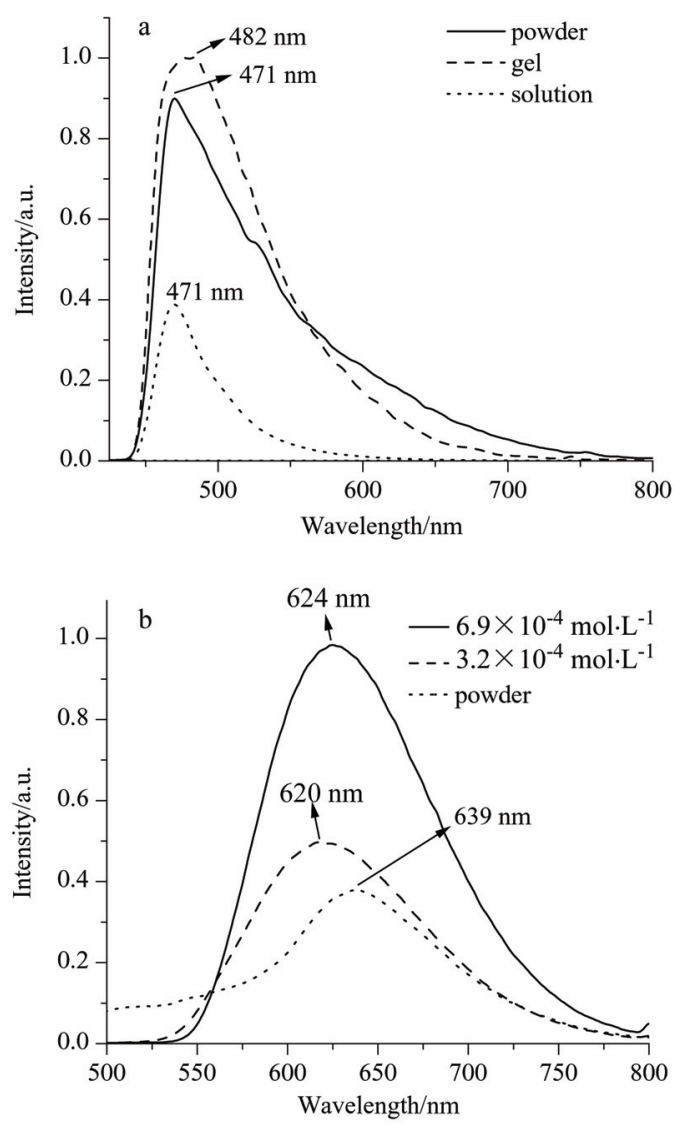

图 2 (a) 1 的 DMSO 溶液 $\left(5.0 \times 10^{-4} \mathrm{~mol} \cdot \mathrm{L}^{-1}, \mathrm{DMSO}\right)$ 、固体粉末和凝 胶 $\left(12.5 \mathrm{mg} \cdot \mathrm{mL}^{-1}\right)$ 的发射光谱 $\left(\lambda_{\mathrm{ex}}=377 \mathrm{~nm}\right.$ )及(b) 2 的 DMSO 稀溶液 $\left(3.2 \times 10^{-4} \mathrm{~mol} \cdot \mathrm{L}^{-1}\right)$ 、饱和溶液 $\left(6.9 \times 10^{-4} \mathrm{~mol} \cdot \mathrm{L}^{-1}\right)$ 和固体的苂光发射 光谱 $\left(\lambda_{\mathrm{ex}}=448 \mathrm{~nm}\right)$

Figure 2 Fluorescent spectra of (a) $\mathbf{1}$ in solution $\left(5.0 \times 10^{-4} \mathrm{~mol} \cdot \mathrm{L}^{-1}\right.$, DMSO), powder and DMSO gel $\left(12.5 \mathrm{mg} \cdot \mathrm{mL}^{-1}, \lambda_{\mathrm{ex}}=377 \mathrm{~nm}\right)$, and (b) 2 in diluted $\left(3.2 \times 10^{-4} \mathrm{~mol} \cdot \mathrm{L}^{-1}\right)$ and saturated $\left(6.9 \times 10^{-4} \mathrm{~mol} \cdot \mathrm{L}^{-1}\right)$ solution of DMSO and the solid of $2\left(\lambda_{\mathrm{ex}}=448 \mathrm{~nm}\right)$

\section{2 两组分在纳米层次的共组装研究}

化合物 1 在 DMSO, DMF, THF 和短链醇中可以形 成凝胶 (辅助材料图 S5) ${ }^{[16]}$, 两组分 $\mathbf{1}+\mathbf{2}$ (物)质的量之比 为 $1: 1$ ) 只能在 DMSO, DMF 和甲醇中形成凝胶(超声处
理), 最低成胶浓度分别为 $6.25,20.0$ 和 $15.0 \mathrm{mg} / \mathrm{mL}$ (表 1). 电子扫描显微镜(SEM)和 CLSM 照片表明 1 在 DMF 中形成短的纤维(辅助材料图 S6), 在 DMSO 中则形成致 密的螺旋纳米纤维(宽约 $40 \mathrm{~nm}$, 图 $3 \mathrm{a}$ 和 $3 \mathrm{~b}$ ). 分子 2 在 DMF 或 DMSO 中能形成棒状的微晶沉淀(辅助材料图 S7). 因此通过 1 和 2 在 DMSO 或 DMF 中的组装就构建 了一种新的凝胶-微晶体系, 从而可以研究该体系中异 种分子间的凝胶和晶化行为. 其中 $\mathbf{1}$ 的 $\mathrm{COOH}$ 和 $\mathbf{2}$ 的苊 唑-N 有可能进行配位作用，从而进行组装. 经过一个简 单的加热-冷却过程, 1 和 2 的混合物仍能形成凝胶, CLSM 图表明, 2 的棒状结构被简单地分散在 $\mathbf{1}$ 的纤维网 络中(图 4a 4c), 并没有如我们预料那样通过分子间的 氢键实现异种分子间的共组装，这可能是由于两种分子 自身具有较强的作用力, 从而只实现了同种分子的自组 装. 有趣的是, 通过超声波预处理 $\left(5 \mathrm{~min}, 0.37 \mathrm{~W} \cdot \mathrm{cm}^{-2}\right.$, $40 \mathrm{kHz}$ ), 然后再加热-冷却 $(\mathrm{S}-\mathrm{H}-\mathrm{C})$, 我们发现 $\mathbf{1}+\mathbf{2}$ (物 质的量比 $1: 0<1 / 2<1: 1)$ 能够很好地实现聚集体的共 组装(图 4d 4f). SEM 和 TEM 照片表明此时形成的纤维 宽度变为 100 $200 \mathrm{~nm}$ (图 3c 和 3d), 这表明 2 分子被很 好地插入到 1 的纤维结构中, 从而导致了纤维变宽.

表 1 化合物 1 及两组分 $(1+2)$ 的成胶性能

Table 1 Gelation properties of $\mathbf{1}$ and $\mathbf{1}+\mathbf{2}$

\begin{tabular}{lcc}
\hline 溶剂 & $\mathbf{1}(6.7 \mathrm{mg} / \mathrm{mL})^{a}$ & $\mathbf{1 + 2}(1: 1)^{b}$ \\
\hline DMSO & $\mathrm{G}$ & $\mathrm{G}(6.25 \mathrm{mg} / \mathrm{mL})$ \\
DMF & $\mathrm{G}$ & $\mathrm{G}(20 \mathrm{mg} / \mathrm{mL})$ \\
THF & $\mathrm{G}$ & $\mathrm{P}$ \\
Methanol & $\mathrm{G}$ & $\mathrm{G}(15 \mathrm{mg} / \mathrm{mL})$ \\
Ethanol & $\mathrm{G}$ & $\mathrm{P}$ \\
Isoprapanol & $\mathrm{G}$ & $\mathrm{P}$ \\
\hline${ }^{a}$ H-C process; ${ }^{b}$ S-H-C process, 括号中为 $\mathbf{1}$ 的最低成胶浓度. G: gel, P: \\
precipitate after S-H-C process.
\end{tabular}
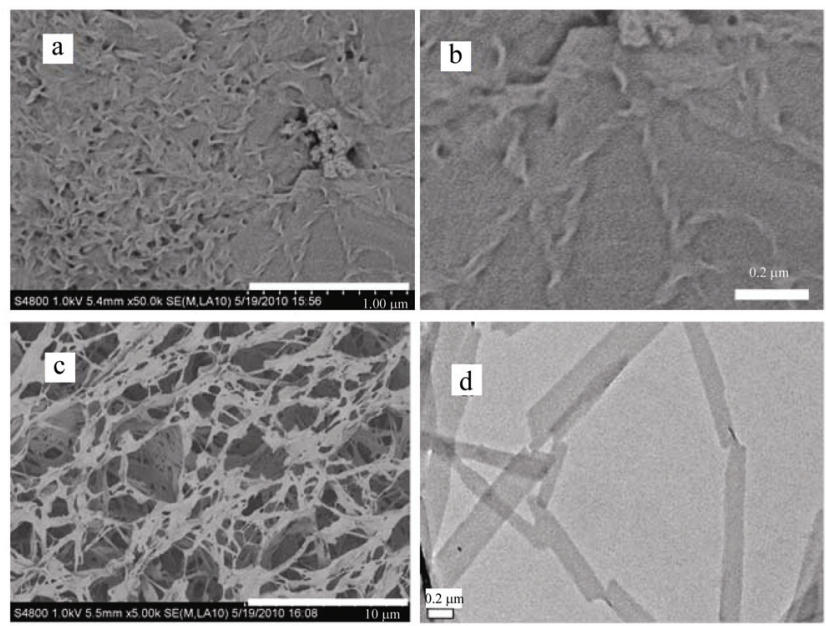

图 $3(\mathrm{a}, \mathrm{b}) \mathbf{1}\left(12.5 \mathrm{mg} \cdot \mathrm{mL}^{-1}\right)$ 在 DMSO 中形成凝胶的 SEM 图片、(c) 1 +2 在 DMSO 中形成凝胶的 SEM 图片和(d) $1+2$ 组装体干凝胶的 TEM 图片

Figure 3 SEM images of xerogels of $\mathbf{1}$ ( $a$ and $b$ ) and $\mathbf{1}+\mathbf{2}$ (c) from DMSO and TEM image of $\mathbf{1}+\mathbf{2}(\mathrm{d})$

$c_{1}: 12.5 \mathrm{mg} \cdot \mathrm{mL}^{-1} ; \mathbf{1}+\mathbf{2}: 12.5 \mathrm{mg} \cdot \mathrm{mL}^{-1}$ of $\mathbf{1}$, molar ratio of $\mathbf{1} / \mathbf{2}=1: 1$ 

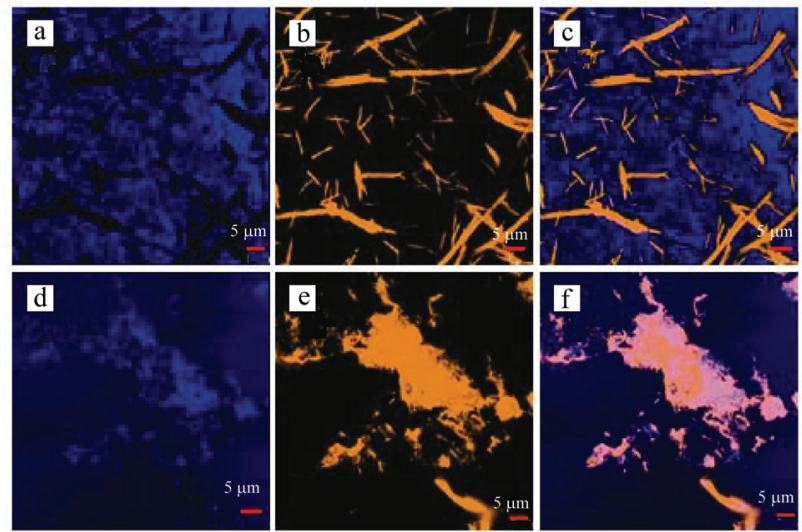

图 4 不同凝胶的 CLSM 图

Figure 4 CLSM images of the gels

$(\mathrm{a} \sim \mathrm{c})$ gel of $\mathbf{1}+\mathbf{2}$ in DMSO with the molar ratio of $\mathbf{1 / 2}=1: 1$ obtained by heating-cooling process; $(\mathrm{d} \sim \mathrm{f})$ gel of $\mathbf{1}+\mathbf{2}$ in DMSO with the molar ratio of $\mathbf{1} / \mathbf{2}=1: 1$ obtained by S-H-C process. The concentration of $\mathbf{1}$ in $\mathrm{a} \sim \mathrm{f}$ is 12.5 $\mathrm{mg} \cdot \mathrm{mL}^{-1}$; for (a) and (d), $\lambda_{\mathrm{ex}}=405 \mathrm{~nm}, \lambda_{\mathrm{em}}=450 \sim 550 \mathrm{~nm}$; for (b) and (e), $\lambda_{\mathrm{ex}}=488 \mathrm{~nm}, \lambda_{\mathrm{em}}=550 \sim 650 \mathrm{~nm}$; (c) and (f) are overlay images of (a) and (b), (d) and (e), respectively

我们进一步通过圆二色(CD)和红外(IR)光谱证实了 $1+2$ 之间存在着共组装现象. 如图 5a 所示: 1 在 DMSO 中形成的凝胶在 $278 \mathrm{~nm}$ 出现了一个负吸收的 $\mathrm{CD}$ 峰, 这 是由于赖氨酸诱导苯环聚集所产生的手性; 经过超声 后, 该峰移动至 $281 \mathrm{~nm}$ 并伴随着强度增强, 再通过一个 加热-冷却过程, 该信号可被修复. 当加入分子 2 , 通过 一个 $\mathrm{H}-\mathrm{C}$ 过程后, CD 信号从-38 下降至 -25 ; 当通过 一个 S-H-C 过程后, CD 信号可以被 $100 \%$ 地淬灭, 这也 表明 2 的嵌入降低了 1 分子间聚集态的宏观手性(1/2 物 质的量比 $1: 1$ ). IR 光谱也证明了类似的情况. 2 的 $\mathrm{C}=\mathrm{O}$ 振动峰在 $1695 \mathrm{~cm}^{-1}, \mathbf{1}$ 的 $\mathrm{C}=\mathrm{O}$ 振动峰在 $1717 \mathrm{~cm}^{-1}$, 是 羧酸二聚的振动峰. 当经过一个 S-H-C 过程后, 这两个 振动峰都消失掉, 在 $1656 \mathrm{~cm}^{-1}$ 处出现了一个新的振动 峰, 预示了 $\mathrm{COOH}$ 和苍唑 $\mathrm{N}$ 间氢键的形成或质子转移 的发生. 分子 1 的 $\mathrm{NH}$ 振动在 $3277 \mathrm{~cm}^{-1}$, 当组装后, 发 生了微弱的蓝移, 证明了 1 同种分子间氢键的减弱. 通 过单独的超声或加热冷却过程, $\mathrm{C}=\mathrm{O}$ 的振动分别在 1705 和 $1660 \mathrm{~cm}^{-1}$, 预示着异种分子间不完整氢键的存 在.

为了深入探讨 $1+2$ 的组装机理, 我们做了 1 和 $1+$ 2 干凝胶的小角散射(SAXS)和粉末衍射(XRD)(图 6). 如 图 6 所示, $5.74 \mathrm{~nm}$ 处的 $d$ 值介于单分子 1 (3.06)和两倍 分子 1 的长度之间, 能初步判断 1 分子间以二聚体为基 本结构单元. 分子 2 在 SAXS 谱图上没有任何峰, XRD 表示它在 DMSO 中则形成了微晶, 大角处规整的数据 表明分子 2 有很好的晶化结构. 当 $\mathbf{1}$ 与 2 经超声共组装 后，在 XRD 模式下峰值和 1 分子的干凝胶类似，这说明 在 $1+2$ 凝胶中, 2 的晶格瓦解, 分子 2 嵌入 1 的纤维结 构中形成共聚体.

通过以上的光谱和结构表征, 我们推断 $\mathbf{1}+\mathbf{2}$ 共组 装机理如图 7 所示. 在 2 加入之前, 分子 1 以分子二聚
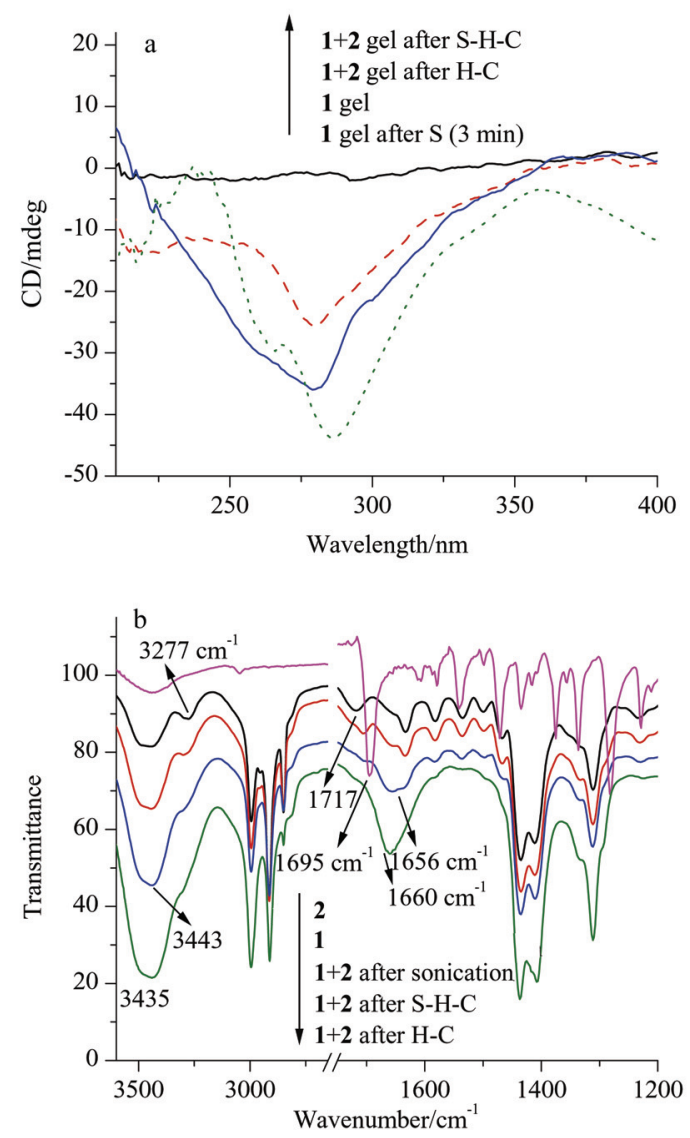

图 5 (a) $\mathbf{1}$ 和 $\mathbf{1}+\mathbf{2}$ 的 $\mathrm{CD}$ 光谱及(b) $\mathbf{1}$ 和 $\mathbf{1}+\mathbf{2}$ 经不同过程的红外光谱 $\left(12.5 \mathrm{mg} \cdot \mathrm{mL}^{-1}\right.$ of $\mathbf{1}$, molar ratio of $\left.\mathbf{1} / \mathbf{2}=1: 1\right)$

Figure 5 (a) $\mathrm{CD}$ and (b) IR spectra of gel $\mathbf{1}, \mathbf{2}, \mathbf{1}+\mathbf{2}, \mathbf{1}+\mathbf{2}$ after sonication, $\mathbf{1}+\mathbf{2}$ from $\mathrm{H}-\mathrm{C}$ process and from $\mathrm{S}-\mathrm{H}-\mathrm{C}$ process $\left(12.5 \mathrm{mg} \cdot \mathrm{mL}^{-1}\right.$ of $\mathbf{1}$, molar ratio of $\mathbf{1} / \mathbf{2}=1: 1$ )

体为基本单元通过分子间氢键和疏水作用进一步组装 为螺旋的纤维. 理论上, 1 分子间氢键的组装模式有两 种: $\alpha-\mathrm{COOH}(\mathrm{C}=\mathrm{O}) /$ amide $(\mathrm{N}-\mathrm{H})($ 模式 A) 或 $\gamma \mathrm{COOH}$ $(\mathrm{C}=\mathrm{O}) /$ amide $(\mathrm{N}-\mathrm{H})$ (模式 B) (辅助材料图 S8). 通过 AM1 半经验法计算可知, 模式 A 因形成了分子间四重 氢键, 所以具有较低能量 $[\alpha-\mathrm{COOH}(\mathrm{C}=\mathrm{O}) / \mathrm{amide}(\mathrm{N}-\mathrm{H})$ (2.26 $\AA$ )和 $\gamma-\mathrm{COOH} / \gamma-\mathrm{COOH}(2.30 \AA)]$, 模式 A 和模式 B 之间的能量差为 0.0115 hartree $\left(30.2 \mathrm{~kJ} \cdot \mathrm{mol}^{-1}\right)$. 当加入 2 之后, 模式 $\mathrm{A}$ 的组装不能提供额外的 $\mathrm{COOH}$ 和苊唑氮 进行配位, 所以通过一个简单的 H-C 过程, 并不能促进 这两种分子间的组装; 超声的刺激对该体系提供了新的 能量, 可以诱导分子 1 间的氢键组装模式发生变化, 迅 速形成热力学不稳定模式 $\mathrm{B}$ 的聚集态，此时模式 B 中分 子 1 裸露的羧基可以和 2 进行分子间配位, 从而进一步 稳定了该聚集态, 促使它们形成了新的均相凝胶体系. 该聚集态非常稳定, 具有高度的热力学稳定性, 通过多 次 H-C 循环, 仍能保持良好的共聚集, 从而形成一种热 力学可逆修复的超声响应的两组分凝胶体系. SAXS 和 $\mathrm{XRD}$ 数据也对这种组装模式转变提供了证据. 

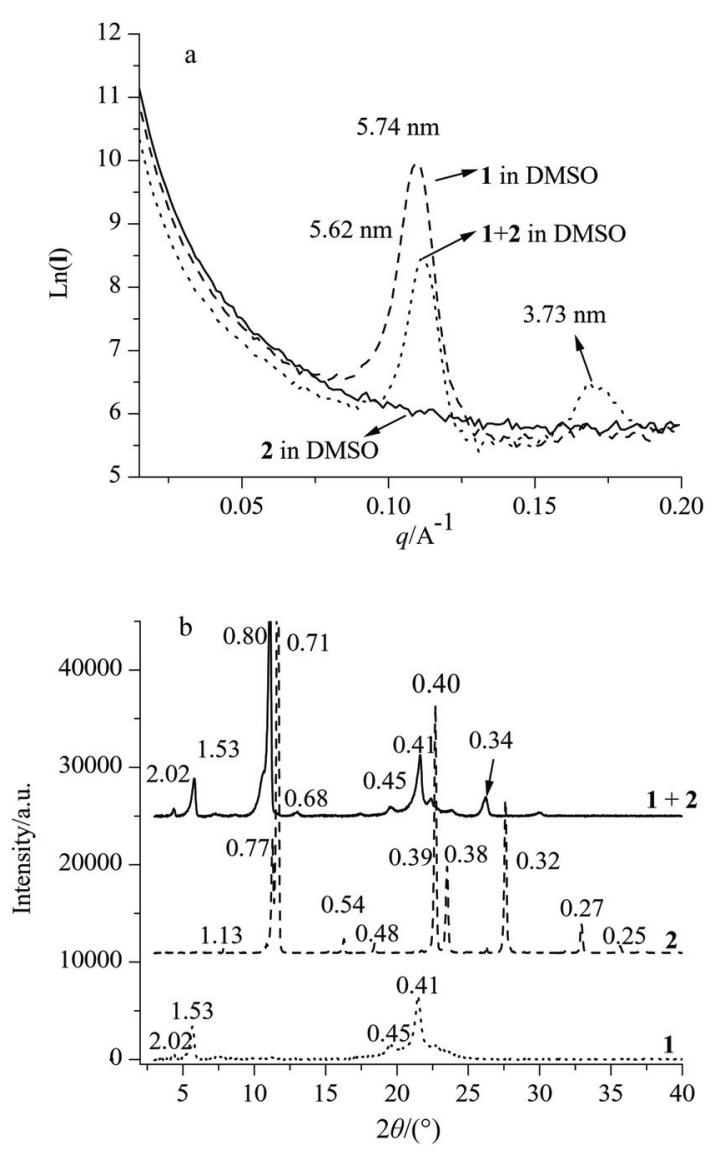

图 $61,1+2$ 和 2 的干凝胶的小角散射和粉末衍射图

Figure 6 (a) SAXS and (b) XRD spectra of $\mathbf{1}$ and $\mathbf{1}+\mathbf{2}$ xerogels and powder of 2 from DMSO

$12.5 \mathrm{mg} \cdot \mathrm{mL}^{-1}$ of $\mathbf{1}$ with the molar ratio of $\mathbf{1} / \mathbf{2}=1: 1$

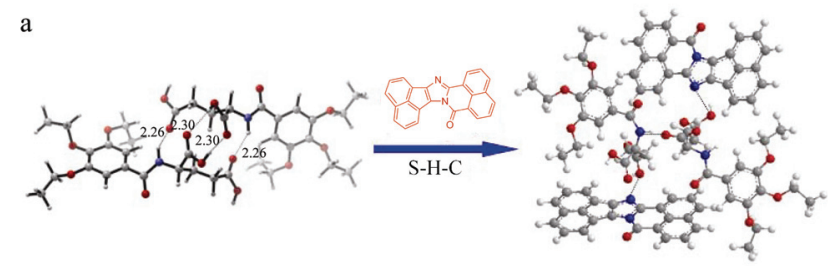

b

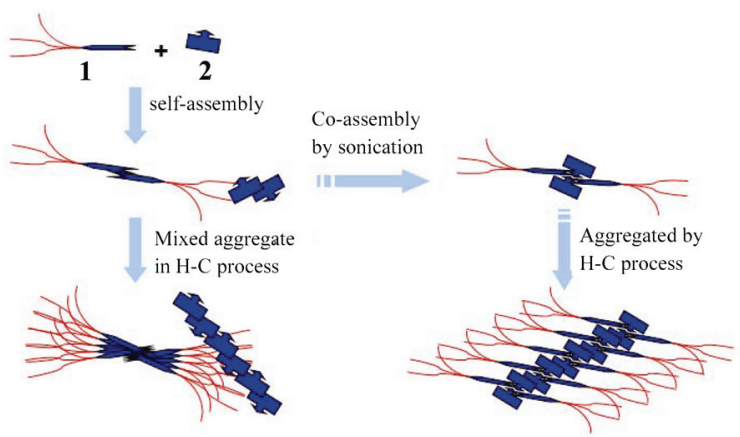

图 7 (a) 计算得到的 1 和 $1+2$ 凝胶的分子聚集模式及(b) $1+2$ 通过 $\mathrm{H}-\mathrm{C}$ 和 S-H-C 过程得到的组装示意图

Figure 7 (a) The calculated hydrogen bonding in $\mathbf{1}$ and $\mathbf{1}+\mathbf{2}$ gel and (b) the prospective aggregation changes of $\mathbf{1}+\mathbf{2}$ in $\mathrm{H}-\mathrm{C}$ and $\mathrm{S}-\mathrm{H}-\mathrm{C}$ process

\section{$2.31+2$ 组装体的多彩荧光发射}

以上结果表明超声为促进两组分或多组分的共组 装提供了一种非常有效的手段. 通过这种组装可获得多 功能或功能协同的软材料. 在本研究中, 我们发现 $\mathbf{1}+\mathbf{2}$ 凝胶的发射光谱可以通过两组分间的能量传递过程进 行有效控制，而这种能量传递过程与两组分的比例息息 相关. 图 $8 \mathrm{a}$ 显示了经 S-H-C 处理的 $\mathbf{1}+\mathbf{2}$ 凝胶中主体分 子 $\mathbf{1}$ (给体)的荧光寿命 $\left(\tau_{\mathrm{D}}\right)$ 及 $\mathbf{1}$ 和 $\mathbf{2}$ 间的能量转化效率 $(E)$ 随不同比例客体分子 2(受体)变化的关系. 从图中可见 能量转移效率随受体比例的增大而增大, 当 $2 / 1$ 物质的 量比 $\geqslant 0.9$ 时, $E$ 值达到 0.74 并保持恒定. 这样, 通过调 节 1 和 2 的比例从 $1: 0$ 到 $1: 1$, 可以获得蓝色、紫色、 粉红、黄色到红色的荧光发射(图 1).

\section{$2.41+2$ 组装体对酸的响应}

进一步研究表明, 2 和 $1+2$ 凝胶可以对酸响应. 当 向 2 的 DMSO 稀溶液 $\left(1.37 \times 10^{-4} \mathrm{~mol} \cdot \mathrm{L}^{-1}\right)$ 中滴加 $\mathrm{HCl}$ 后，它的发射波长会发生蓝移，并且荧光增强，同时伴 随着颜色由红色变为橙色, 这是由于杂环上的 $\mathrm{N}$ 质子化 引起了整个杂环离域轨道的电子云密度降低(图 8b). 我 们也发现, $1+2$ 的凝胶也可以对酸响应, 并且呈现出宏 观的相态及发射光谱的变化. 例如: 当 $\mathrm{pH}=2$ 时, 会引 起凝胶 - 溶胶的转变, 利用不同配比的 $\mathbf{1}+\mathbf{2}$ 凝胶 $\left(c_{1}\right.$ : $12.5 \mathrm{mg} \cdot \mathrm{mL}^{-1}$ ) 可以得到不同发射波长的溶胶. 当 $\mathbf{1} / \mathbf{2}$ 的 物质的量之比为 $1: 0.02$ 或 $1: 0.09$ 时, 发射光分别由紫 色和桃红色变为白色(图 $8 \mathrm{c}, 8 \mathrm{~d}$ ); 当 $\mathbf{1 / 2}$ 的物质的量之比 为 $1: 0.18$ 或 $1: 0.66$ 时, 发射光谱由橙色变为亮黄色或 从红色变亮红色(图 $8 \mathrm{e}, 8 \mathrm{f}$ ). 虽然它们在酸的存在下都 变为了溶胶, 但是 CLSM 图证实, 这种两组分在酸的诱 导下形成了直径为 $1 \sim 3 \mu \mathrm{m}$ 的囊泡结构(辅助材料图 $\mathrm{S} 10$ ), 蓝光和白光有较好的重叠(图 $9 a \sim 9 c$ ), 这可能是 由于质子化的 2 为囊泡的形成提供了亲水部分. 当加入 $\mathrm{FeCl}_{3}$ (0.5 equiv.)作为一种路易斯酸时, 也能诱导 $\mathbf{1}+\mathbf{2}$ 组装体从纤维结构向球形结构的转变 (图 9d 9f), 形成 直径为 $0.6 \sim 1 \mu \mathrm{m}$ 均匀分散的囊泡(辅助材料图 S11). 我 们还发现这种组装与三种物质的加入顺序有关: 如果先 把 2 质子化, 然后再加入 $\mathbf{1}$, 通过一个 S-H-C 过程, 并不 能实现它们聚集体之间的共组装.

研究还表明, 向酸诱导的 $\mathbf{1}+\mathbf{2}$ 溶胶中加入碱可以 使其恢复为凝胶态. 例如：把等物质的量的三乙胺加入 至酸化的 $1+2$ 溶胶中, 然后经过 $\mathrm{H}-\mathrm{C}$ 过程, 该溶胶可以 逐渐恢复为凝胶态(辅助材料图 S12). 需要注意的是, 该恢复的凝胶并不稳定, 轻微晃动, 凝胶排除溶剂并发 生塌陷. CLSM 图表明, 此时 $\mathbf{1}+\mathbf{2}$ 和三乙胺的盐酸盐形 成了一种杂化的核壳结构(不发光的块状三乙胺的盐酸 盐作为核, 同时发射红光和蓝光的 $\mathbf{1}+\mathbf{2}$ 形成的纤维为 壳，辅助材料图 S13). 当用水洗涤，该盐酸盐的核可以 被水洗掉, 此时的干凝胶可以重新在 DMSO 中形成 

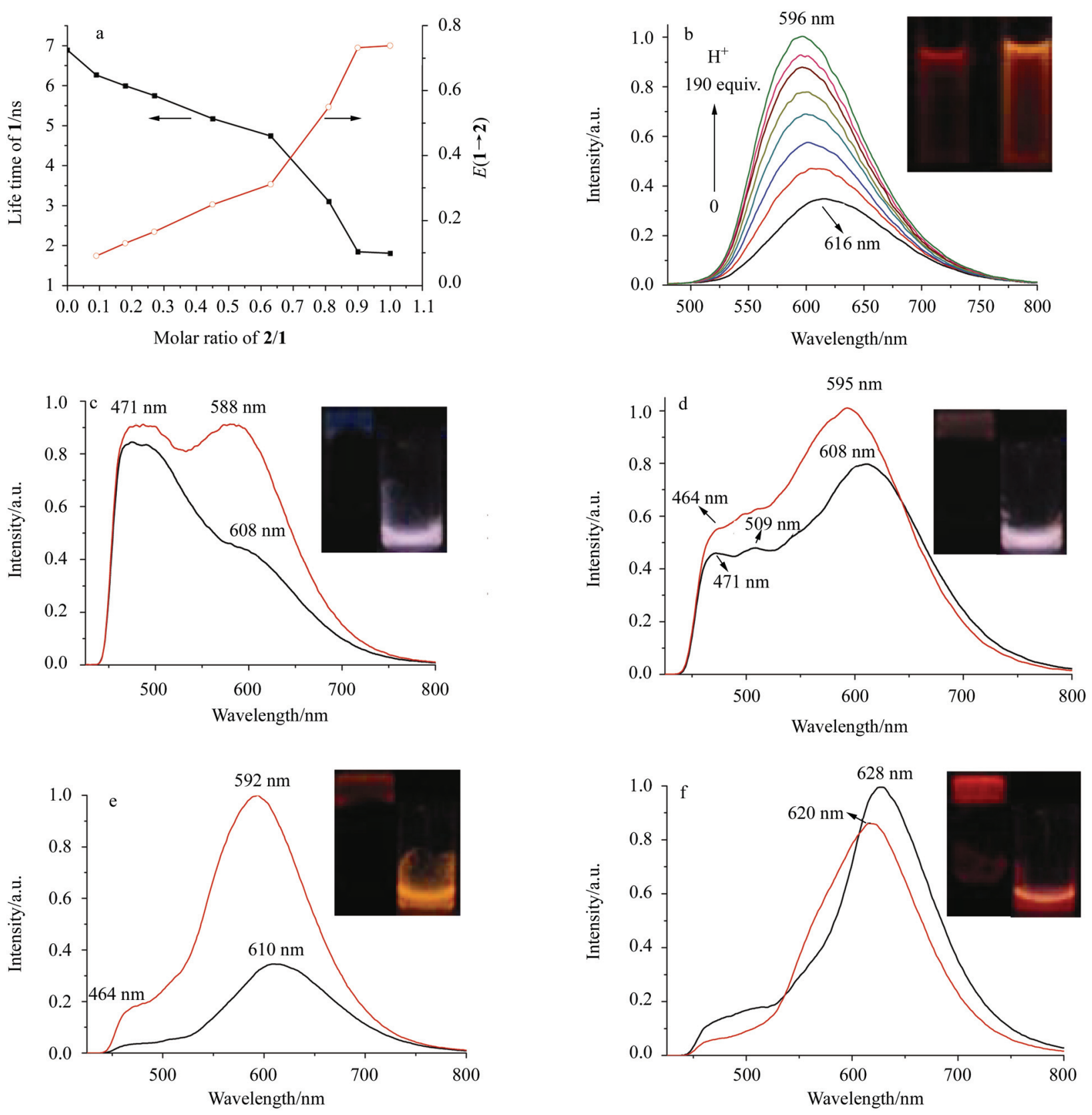

图 8 (a) 凝胶 $\mathbf{1}$ 的荧光寿命及 $\mathbf{1}+\mathbf{2}$ 凝胶中不同配比下 $\mathbf{1}$ 和 $\mathbf{2}$ 之间的能量传递效率(DMSO 溶剂)、(b) $\mathbf{2}$ 的溶液对酸响应的荧光滴定曲线 $\left(\lambda_{\mathrm{ex}}=448\right.$ $\mathrm{nm}) ;(\mathrm{c} \sim \mathrm{f})$ 不同配比的 $\mathbf{1}+\mathbf{2}$ 凝胶的荧光光谱(凝胶, 黑线)及它们对酸响应后的荧光光谱变化(溶胶, 红线) (插图为盐酸加入前后的荧光对比)

Figure 8 (a) The life time of $\mathbf{1}$ and ET efficiency in $\mathbf{1}+\mathbf{2}$ gels with different molar ratio of $\mathbf{2} / \mathbf{1}$ in DMSO, (b) the emission of $\mathbf{2}$ in DMSO solution (1.37 $\left.\times 10^{-4} \mathrm{~mol} \cdot \mathrm{L}^{-1}\right)$ with the addition of different amount of $\mathrm{HCl}\left(\lambda_{\mathrm{ex}}=448 \mathrm{~nm}\right)$ and $(\mathrm{c} \sim \mathrm{f})$ fluorescent spectra of $\mathbf{1}+\mathbf{2}$ gels with the different molar ratio of $\mathbf{1} / \mathbf{2}$ (black line) and the changes when treated with acid to $\mathrm{pH}=2$ (sols, red line) (the insets are the images before and after addition of $\mathrm{HCl}$ ) (c) $1: 0.02$, (d) $1: 0.09$, (e) $1: 0.18$ and (f) $1: 0.66$. The concentration of 1 in all the gel samples is $12.5 \mathrm{mg} \cdot \mathrm{mL}^{-1}$

稳定的凝胶. 总而言之, 该 $\mathbf{1}+\mathbf{2}$ 两组分凝胶可以对酸作 出响应，进一步丰富其苂光光谱色彩，同时伴随着微观 形貌和凝胶-溶胶等宏观状态的显著变化. 通过碱的进 一步调控, 可恢复其凝胶体系. 该传感体系展示出良好 的光学和形态的可逆性. 本研究提供了一种全新的多组 分聚集体系用于可视化传感的新方法.

\section{3 结论}

本工作中, 我们发现超声和热的协同作用可以有效
调控两种不同分子间的组装行为, 促使异种分子由相分 离态的自组装变为分子间的共组装. 我们所选择的蓝光 和红光两种发射波长迥异的分子可以使我们很有效地、 直接地在共聚焦显微镜下观测到两种分子聚集体的组 装行为. 同时, 通过两组分间比例的精确调控, 可以有 效地调控该两组分凝胶的发光. 该两组分体系可以对质 子酸或金属离子作出响应，其苂光发射可以进一步被酸 来调控. 当被酸或金属离子刺激时, 该两组分的微观形 貌可以实现从纤维到囊泡的转变, 并伴随着溶胶-凝胶 

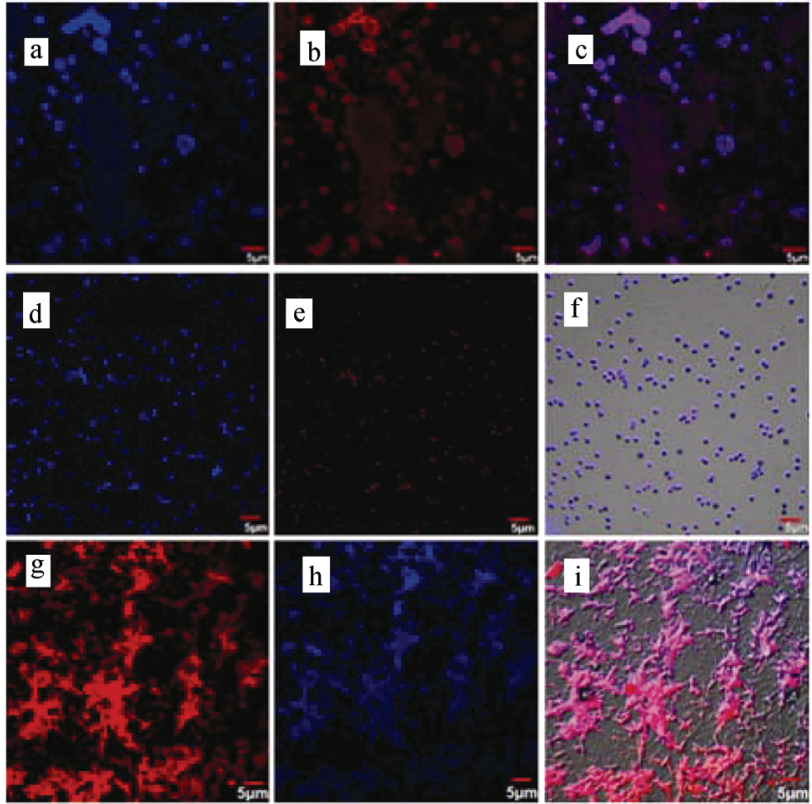

图 $91+2$ 凝胶对酸响应的 CLSM 图

Figure 9 CLSM images of $\mathbf{1}+\mathbf{2}$ responding to acid

$(\mathrm{a} \sim \mathrm{c}) \mathbf{1}+\mathbf{2}$ gel with the molar ratio of $1: 1$ treated with 1 equiv. of $\mathrm{HCl}$ in DMSO; $(\mathrm{d} \sim \mathrm{f}) \mathbf{1}+\mathbf{2}$ gel in DMF $\left(40 \mathrm{mg} \cdot \mathrm{mL}^{-1}\right.$ of $\mathbf{1}$, with the molar ratio of $\mathbf{1} / \mathbf{2}$ $=1: 0.18)$ treated with $\mathrm{FeCl}_{3}(0.5$ equiv. of $\mathbf{1}) ;(\mathrm{g} \sim \mathrm{i})$ the reversed $\mathbf{1}+\mathbf{2}$ gel after acid/base process and washed by water. The concentration of $\mathbf{1}$ in $\mathrm{a} \sim \mathrm{i}$ is $12.5 \mathrm{mg} \cdot \mathrm{mL}^{-1}$; for (a), (d) and (g), $\lambda_{\mathrm{ex}}=405 \mathrm{~nm}, \lambda_{\mathrm{em}}=450 \sim 550 \mathrm{~nm}$; for (b), (e) and (h), $\lambda_{\mathrm{ex}}=488 \mathrm{~nm}, \lambda_{\mathrm{em}}=550 \sim 650 \mathrm{~nm}$; (c), (f) and (i) are overlay images of (a) and (b), (d) and (e), (g) and (h), respectively; bright field is additionally plus to $\mathrm{f}$

的宏观形貌变化; 进一步地, 通过加入碱可以诱导该溶 胶还原为凝胶, 实现宏观相态的可逆变化. 这种具有多 重功能并易于调控的两组分凝胶在可视化分子识别、 控制缓释、刺激响应和功能记忆材料等领域具有广泛的 潜在应用.

\section{4 实验部分}

\section{1 合成和表征}

1 的合成在我们过去的工作中已发表 ${ }^{[16]}$. 范醌 $(0.3$ $\mathrm{g}, 2.2 \mathrm{mmol}$ ) 和 $4 \mathrm{~g}$ 醋酸胺 $(65 \mathrm{mmol}$ ) 在 $30 \mathrm{~mL}$ 冰醋酸中 加热回流. 冷却后, 沉淀经 $\mathrm{Al}_{2} \mathrm{O}_{3}$ 柱层析[洗脱液: $V($ 二 氯甲烷)： $V$ (石油醚 $)=5 ： 1$ ] 得到红色固体 2 . 产率 $52 \%$, m.p. $>250{ }^{\circ} \mathrm{C} .{ }^{1} \mathrm{H}$ NMR $\left(\mathrm{CDCl}_{3}, 400 \mathrm{MHz}\right) \delta: 8.88(\mathrm{~d}, J=$ $7.2 \mathrm{~Hz}, 1 \mathrm{H}), 8.77(\mathrm{~d}, J=7.2 \mathrm{~Hz}, 1 \mathrm{H}), 8.42(\mathrm{~d}, J=6.8 \mathrm{~Hz}$, $1 \mathrm{H}), 8.32(\mathrm{~d}, J=8 \mathrm{~Hz}, 1 \mathrm{H}), 8.09 \sim 8.07(\mathrm{~m}, 2 \mathrm{H}), 7.85(\mathrm{t}$, $J=7.2 \mathrm{~Hz}, 3 \mathrm{H}), 7.79$ (t, $J=8 \mathrm{~Hz}, 1 \mathrm{H}), 7.68 \sim 7.62(\mathrm{~m}$, $2 \mathrm{H}$ ); HRMS (ESI) calcd for $\mathrm{C}_{24} \mathrm{H}_{13} \mathrm{~N}_{2} \mathrm{O}: 345.1005[\mathrm{M}+$ $\mathrm{H}^{+}$], found 345.1028 .

\section{2 方法和手段}

${ }^{1} \mathrm{H}$ NMR 在 Mercuryplus $400 \mathrm{MHz}$ 上获得, $\mathrm{CDCl}_{3}$ 为 溶剂, 以 TMS 为内标. MALDI-TOF 在岛津 AXIMACFRPLVS 型质谱仪上获得. 熔点在 XT4-100A 型仪器 上测得. 傅立叶变换红外光谱 (FT-IR) 在岛津
IRPRESTIGE-21 型红外光谱上测得, 分为干凝胶和湿 凝胶两种, 干凝胶是由 $\mathrm{KBr}$ 压片, 除说明外红外都是湿 凝胶数据. 干凝胶的扫描电镜 (SEM) 图片在岛津的 SSX-550 和日立的 FE-SEM S-4800 场发射扫描电镜上测 得，样品通过湿凝胶冷冻干燥然后喷金制得. 透射电镜 (TEM) 在 JEOL JEM2011 的仪器上测得, 把湿凝胶样品 滴在铜网上冷冻干燥 $48 \mathrm{~h}$ 得干凝胶样品. 小角衍射在布 鲁克公司的 nanostar U SAXS 型小角衍射仪上测得, X射 线源为 AXS D8 $(\mathrm{Cu}$ target; $\lambda=0.1542 \mathrm{~nm}, 40 \mathrm{kV} \times 50$ $\mathrm{mA}$ ). 超声波仪的型号是 KQ-500DB，最大功率 $100 \mathrm{~W}$, $40 \mathrm{kHz}$, 中国昆山超声仪器有限公司.

CLSM 是在 OLYMPUS IX81 配备 60X 油镜, 利用 不同的波长激发, 分别收集 $450 \sim 550$ 和 $550 \sim 650 \mathrm{~nm}$ 区 间的发射. 在载玻片上制备得到干凝胶作为测试样品.

\section{3 凝胶实验}

T-gel: 通过加热 $120{ }^{\circ} \mathrm{C}$ 到固体完全溶解, 然后冷 却至室温(H-C process); S-H-C process: 混合物加热至 $120{ }^{\circ} \mathrm{C}$. 然后放入恒温水浴 $\left(25{ }^{\circ} \mathrm{C}\right)$ 中超声 $5 \mathrm{~min}$, 然后 该较差的凝胶通过进一步加热冷却得到稳定凝胶.

\section{References}

[1] (a) Partridge, K. S.; Smith, D. K.; Dykes, G. M.; McGrail, P. T. Chem. Commun. 2001, 319; (b) Hirst, A. R.; Smith, D. K.; Harrington, J. P. Chem.-Eur. J. 2005, 11, 6552; (c) Hirst, A. R.; Smith, D. K.; Feiters, M. C.; Geurts, H. P. M. Langmuir 2004, 20, 7070; (d) Hirst, A. R.; Smith, D. K. Langmuir 2004, 20, 10851; (e) Hirst, A. R.; Smith, D. K.; Feiters, M. C.; Geurts, H. P. M. Chem.-Eur. J. 2004, 10, 5901; (f) Hirst, A. R.; Smith, D. K.; Feiters, M. C.; Geurts, H. P. M.; Wright, A. C. J. Am. Chem. Soc. 2003, 125, 9010; (g) Simalou, O.; Xue, P.; Lu, R. Tetrahedron Lett. 2010, 51, 3685; (h) Suzuki, M.; Saito, H.; Hanabusa, K. Langmuir 2009, 25, 8579; (i) Zhou, Y.; Xu, M.; Yi, T.; Xiao, S.; Zhou, Z.; Li, F.; Huang, C. Langmuir 2007, 23, 202; (j) Yi, T.; Sada, K.; Sugiyasu, K.; Hatano, T.; Shinkai, S. Chem. Commun. 2003, 344.

[2] (a) Ajayaghosh, A.; Chithra, P.; Varghese, R. Angew. Chem., Int. Ed. 2007, 46, 230; (b) Suzuki, M.; Yumoto, M.; Shirai, H.; Hanabusa, K. Chem.-Eur. J. 2008, 14, 2133.

[3] (a) Ajayaghosh, A.; Praveen, V. K.; Vijayakumar, C.; George, S. J. Angew. Chem., Int. Ed. 2007, 46, 6260; (b) Shu, T.; Wu, J.; Lu, M.; Chen, L.; Yi, T.; Li, F.; Huang, C. J. Mater. Chem. 2008, 18, 886; (c) Bao, C.; Lu, R.; Jin, M.; Xue, P.; Tan, C.; Liu, G.; Zhao, Y. Org. Biomol. Chem. 2005, 3, 2508; (d) Babu, P.; Sangeetha, N. M.; Vijaykumar, P.; Maitra, U.; Rissanen, K.; Raju, A. R. Chem.-Eur. J. 2003, 9, 1922.

[4] (a) Liao, P.; Langloss, B. W.; Johnson, A. M.; Knudsen, E. R.; Tham, F. S.; Julian, R. R.; Hooley, R. J. Chem. Commun. 2010, 46 4932; (b) Huang, S.; Li, X.; Shi, X.; Hou, H.; Fan, Y. T. J. Mater. Chem. 2010, 20, 5695; (c) Valente, C.; Choi, E.; Belowich, M. E.; Doonan, C. J.; Li, Q. W.; Gasa, T. B.; Botros, Y. Y.; Yaghi, O. M.; Stoddart, J. F. Chem. Commun. 2010, 46, 4911; (d) Weng, W.; Beck, J. B.; Jamieson, A. M.; Rowan, S. J. J. Am. Chem. Soc. 2006, 128, 11663; (e) Paoli, G. D.; Džolic, Z.; Rizzo, F.; Cola, L. D.; Vögtle, F.; Müller, W. M.; Richardt, G.; Žinic, M. Adv. Funct. Mater. 2007, 17, 821; (f) Hui, J. K.-H.; Yu, Z.; MacLachlan, M. J. Angew. Chem., Int. Ed. 2007, 46, 7980; (g) George, M.; Funkhouser, G. P.; Terech, P. R.; Weiss, G. Langmuir 2006, 22, 7885.

[5] (a) Molla, M. R.; Das, A.; Ghosh, S. Chem.-Eur. J. 2010, 16, 10084 ; (b) Das, R. K.; Banerjee, S.; Raffy, G.; Del Guerzo, A.; Desvergne, J.-P.; Maitra, U. J. Mater. Chem. 2010, 20, 7227; (c) Friggeri, A.; Gronwald, O.; van Bommel, K. J. C.; Shinkai, S.; Reinhoudt, D. N. J. Am. Chem. Soc. 2002, 124, 10754; (d) Rao, K. V.; Jayaramulu, K.; Maji, T. K.; George, S. J. Angew. Chem., Int. Ed. 2010, 49, 4218. 
[6] (a) Wang, W. C.; Du, C.; Bi, H.; Sun, Y. H.; Wang, Y.; Mauser, C.; Como, E. D.; Fuchs, H.; Chi, L. F. Adv. Mater. 2010, 22, 2764; (b) Nalluri, S. K. M.; Ravoo, B. J. Angew. Chem., Int. Ed. 2010, 49, 5371; (c) Mahesh, S.; Thirumalai, R.; Yagai, S.; Kitamura, A.; Ajayaghosh, A. Chem. Commun. 2009, 5984; (d) Chen, Q.; Zhang, D.; Zhang, G.; Yang, X.; Feng, Y.; Fang, Q.; Zhu, D. B. Adv. Funct. Mater. 2010, 20, 3244; (e) Shumburo, A.; Biewer, M. C. Chem. Mater. 2002, 14, 3745; (f) Li, J. L.; Liu, X. Y. Adv. Funct. Mater. 2010, 20, 3196; (g) Wang, C.; Chen, Q.; Sun, F.; Zhang, D.; Zhang, G.; Huang, Y.; Zhao, R.; Zhu, D. J. Am. Chem. Soc. 2010, 132, 3092 .

[7] (a) Mukhopadhyay, P.; Iwashita, Y.; shirakawa, M.; Kawano, S.; Fujita, N.; Shinkai, S. Angew. Chem., Int. Ed. 2006, 45, 1592; (b) Ajayaghosh, A.; Varghese, R.; Mahesh, S.; Praveen, V. K. Angew. Chem., Int. Ed. 2006, 45, 7729; (c) Palmans, A. R. A.; Meijer, E. W. Angew. Chem., Int. Ed. 2007, 46, 8948.

[8] (a) Naota, T.; Koori, H. J. Am. Chem. Soc. 2005, 127, 9324; (b) Wang, C.; Zhang, D. Q.; Zhu, D. B. J. Am. Chem. Soc. 2005, 127, 16372; (c) Paulusse, J. M. J.; Sijbesma, R. P. Angew. Chem., Int. Ed. 2006, 45, 2334; (d) Bardelang, D. Soft Matter 2009, 5, 1969; (f) Cravotto, G.; Cintas, P. Chem. Soc. Rev. 2009, 38, 2684.
[9] Richards, W. T.; Loomis, A. L. J. Am. Chem. Soc. 1927, 49, 3086.

[10] Donaldson, D. J.; Farrington, M. D.; Kruus, P. J. Phys. Chem. 1979, 83,3130

[11] Petrier, C.; Jeunet, A.; Luche, J.-L.; Reverdy, G. J. Am. Chem. Soc. 1992, 114, 3148 .

[12] Kitazume, T.; Ishikawa, N. J. Am. Chem. Soc. 1985, 107, 5186.

[13] (a) Wu, J.; Yi, T.; Shu, T.; Yu, M.; Zhou, Z.; Xu, M.; Zhou, Y.; Zhang, H.; Han, J.; Li, F.; Huang, C. Angew. Chem., Int. Ed. 2008, 47, 1063; (b) Wu, J.; Yi, T.; Xia, Q.; Zou, Y.; Liu, F.; Dong, J.; Shu, T.; Li, F.; Huang, C. Chem.-Eur. J. 2009, 15, 6234.

[14] Yu, X.; Liu, Q.; Wu, J.; Zhang, M.; Cao, X.; Zhang, H.; Wang, Q.; Chen, L.; Yi, T. Chem.-Eur. J. 2010, 16, 9099.

[15] Anderson, K. M.; Day, G. M.; Paterson, M. J.; Byrne, P.; Clarke, N.; Steed, J. W. Angew. Chem., Int. Ed. 2008, 47, 1058.

[16] Chen, L.; Wu, J.; Shu, T.; Xu, M.; Zhang, M.; Yi, T. Langmuir 2009, 25, 8434 .

[17] Zhang, M.; Yu, M.; Li, F.; Zhu, M.; Li, M.; Gao, Y.; Li, L.; Liu, Z.; Zhang, J.; Zhang, D.; Yi, T. Huang, C. J. Am. Chem. Soc. 2007, 129, 10322 .

[18] (a) Weiss, M. J. Am. Chem. Soc. 1952, 74, 5193; (b) White, D. M. J. Org. Chem. 1970, 35, 2452.

(Zhao, C.) 